\title{
Comparison of different conditions for accelerated ageing of small molecule organic solar cells
}

\author{
Martin Hermenau*a, Karl Leo ${ }^{\text {a }}$, Moritz Riede ${ }^{\mathrm{a}}$ \\ anstitut für Angewandte Photophysik, Technische Universität Dresden, 01069 Dresden, Germany
}

\begin{abstract}
Besides efficiency and cost, lifetime is another important factor for the commercialisation of small molecule organic solar cells. To quickly achieve results one has to perform accelerated measurements. Thus, knowledge about accelerating factors is necessary to relate these results with measurements under real working conditions. Here, we compare different conditions for accelerated lifetime measurements of organic solar cells. The investigated p-i-n-devices contain a bulk heterojunction of Zinc-Phthalocyanine $(\mathrm{ZnPc})$ and the fullerene $\mathrm{C} 60$ as photoactive materials. Doped layers of a large triarylamine-based amorphous wide gap material (Di-NPB) and C60 are used as hole and electron transport layer, respectively. For all devices, the IV characteristics are recorded during the entire measuring time.

Unencapsulated solar cells show a rapid degradation due to the strong impact of atmospheric gases like oxygen or water vapour. Lifetimes $\left(\mathrm{t}_{80}\right)$ of 43 to 110 hours are observed. Devices illuminated by blue light show a faster degradation than those exposed to red light. Additionally, the degradation is further accelerated when the intensity of blue light is increased. The comparison of external quantum efficiency measurements performed before and after ageing verifies that the used photoactive materials are stable. The intensity has the largest influence on degradation dynamics. Our results for solar cells illuminated by white light LEDs show that at intensities up to $100 \mathrm{~mW} / \mathrm{cm}^{2}$ the power conversion efficiency increases with time. This effect was observed over nearly 2000 hours of operation. An intensity of more than five suns is required to reduce the efficiency of our solar cells with time. This reduction is mainly driven by losses in the Fill Factor and a slight decrease of short circuit current density. Nevertheless, extrapolated lifetimes of up to 5000 hours are still observed.
\end{abstract}

Keywords: organic solar cell, small molecule, lifetime, degradation, stability

\section{INTRODUCTION}

\subsection{Organic Solar Cells}

Throughout the last years an increasing effort was made in the field of Organic Semiconductors with the common goal of cheap and flexible electronics. As one part of this field Organic Solar Cells emerged quickly. In this area, two different material types are typically differentiated: Polymer materials, which are normally processed by wet chemical processes and, in contrast, materials which consist of small molecules, that are commonly fabricated in vacuum evaporation systems. For both types of organic solar cells, remarkable efficiency values of $7.9 \%$ on small areas of $0.047 \mathrm{~cm}^{2}$ for polymer materials and $6.1 \%$ on an area of more than $1 \mathrm{~cm}^{2}$ for small molecule materials were achieved. ${ }^{1}$ Beside efficiency and cost, lifetime is the third important factor which plays a key role in the commercialization of these devices. Considering the cost per $\mathrm{kWh}$, it is clear that doubling the lifetime has the same effect as doubling the efficiency.

*martin.hermenau@iapp.de; phone +49 351 46339189; fax +49 35146337065 ; www.iapp.de/iapp/agruppen/osol/

Organic Photonics IV, edited by Paul L. Heremans, Reinder Coehoorn, Chihaya Adachi, Proc. of SPIE Vol. 7722, 77220K - @ 2010 SPIE · CCC code: 0277-786X/10/\$18 - doi: 10.1117/12.853834 


\subsection{Ageing Studies on Organic Solar Cells}

Most literature on organic solar cells focuses on improving the efficiency and exploring the fundamental principles of related materials. Only a few publications reported on degradation studies and showed detailed degradation pathways. Most of them concentrated on polymer materials such as P3HT, PCBM or MEH-PPV. ${ }^{6}$

Regarding small molecule organic solar cells, only a minor part of lifetime studies were published in the recent years. Some of them only report so called shelf-lifetimes, which simply means that the solar cells were stored in the dark for a certain time. ${ }^{7-9}$ These solar cells as well as some others only showed poor lifetimes in the range of a few up to several hundred hours, mostly with direct contact to air. ${ }^{10-12}$ Iso far only Heliatek GmbH (Dresden, Germany) reported on a multi-layer tandem cell with doped transport layer and an extrapolated lifetime of 9800h, unfortunately without details about the device structure. ${ }^{13}$ This device was glass-glass encapsulated and showed therefore an excellent intrinsic stability. The degradation experiment was done at about $50^{\circ} \mathrm{C}$ and the intensity of nearly two suns, produced by a halogen lamp. Another study on encapsulated small molecule organic solar cells by Lessmann et al. showed detailed results of experiments, where the ageing of solar cells was caused by oxidation reactions of $\mathrm{C} 60 .^{14}$

The problem of all these reports is that there do not exist standard conditions for lifetime reports. Therefore, multiple parameters like temperature, encapsulation, humidity, light intensity, and light spectrum are different for each study. This makes it nearly impossible to compare different reports and the observed solar cells.

For achieving quick results, so called Accelerated Life Testing (ALT) is used. With this method, the degradation speed is enhanced by applying overstress conditions. Parameters that are expected to accelerate organic solar cell degradation need to be easily controlled. For example, this can be the temperature, the light intensity as well as the light spectrum.

Again, only a small number of reports that present acceleration factor for degradation of organic photovoltaic devices exists. Schuller et al. reported on a ten times faster degradation of glass-glass encapsulated polymer solar cells by increasing the temperature from $25^{\circ} \mathrm{C}$ to $80^{\circ} \mathrm{C} .{ }^{15}$ The work of Gevorgyan et al. show a series of lifetime measurements with different temperatures in the range from $25^{\circ} \mathrm{C}$ to $83^{\circ} \mathrm{C}$, resulting in acceleration factors up to 6.25 in ambient atmosphere. ${ }^{16}$ In the absence of oxygen and water vapour, even lower acceleration factors between 0.65 and 1.4 were observed.

The minor number of reports on accelerated lifetime test on organic solar cells shows that there is lot of work to do, especially considering that the first commercial products are already available. ${ }^{17}$

\section{EXPERIMENTAL}

\subsection{Device preparation}

All presented solar cells are fabricated on a commercially available glass-substrate (Thin Film Devices, USA) in an ultra-high vacuum evaporation system (Kurt J. Lesker Co., U.K.). The substrates have a 100nm thick layer of indium-tinoxide, a transparent conductor. During the evaporation of organic materials and metals a pressure of $10^{-6}$ to $10^{-8} \mathrm{mbar}$ is typical.

On the ITO-coated glass substrate, 30nm of p-doped N.N'-diphenyl-N.N'-bis(4'-(N.N-bis(naphth-1-yl)-amino)-biphenyl4-yl)-benzidine (DiNPB) form typically the first layer of the p-i-n structure. ${ }^{2}$ For doping we use the material NDP2 (Novaled, Germany) with a concentration of 3 to $4 \mathrm{wtl} \%{ }^{3}$ As already mentioned, the observed solar cells use Zinc Phthalocyanine ( $\mathrm{ZnPc}$ ) and $\mathrm{C} 60$ in a 30nm thick bulk heterojunction. The blend ratio is 1:1 or 2:1. As additional active layer, $30 \mathrm{~nm}$ C60 can contribute to the photo current due to the materials high exciton diffusion length of around $40 \mathrm{~nm}^{4}$ On top of these active layers a n-doped C60 with a thickness of 30nm is used as electron transport material. For a high conductivity we use NDN1 (Novaled, Germany). ${ }^{5}$ As top contact 100nm Aluminium are evaporated. To ensure high material pureness all organic materials are sublimated at least twice. 
The following structures of investigated devices are used for the observations reported in this paper. The numbers in brackets represent thicknesses in nanometres and doping in weight percent or mixing ratio respectively.

ITO/ NDP2 (1) / p-DiNPB (30, 4wt\% (device A) or 3wt\% (device B) / ZnPc:C60 (30, 1:1 (device A) or 2:1 (device B) / C60 (30) / n-C60 (30, 2wt \%) / Al (100)

After preparation, all devices are encapsulated by laying a glass lid over the organic layer and sealing of the edges was done with an UV-curable epoxy glue (Nagase XNR5516Z-L). In a cavity we place a getter material to avoid permeating water vapour from getting in contact to the organics. Both processes are carried out under a nitrogen atmosphere.

Due to the used shadow mask and the pre-structured substrates, we have four identical devices on each substrate which gives us the flexibility to employ different ageing conditions easily.

\subsection{Characterisation}

For the initial characterisation directly after production a sun simulator (Optopolymer 16S-150V.3, Germany) is used. The electrical measurements are done with a source measurement unit (Keithley 2400). To secure comparability, the intensity of the sun simulator was mismatch-corrected. ${ }^{20}$

For the determination of the External Quantum Efficiency a self-made setup is used. This setup uses the lock-in technique (lock in amplifier Signal recovery 4262) and bias illumination from a Xe lamp. For a stepwise monochromatic illumination a Cornerstone 260 monochromator is used. In this work we will correct each value of $\mathrm{J}_{\mathrm{SC}}$ and the efficiency $\eta$ to the intensity of one sun $\left(100 \mathrm{~mW} / \mathrm{cm}^{2}\right)$ an by the mismatch factor.

\subsection{Ageing studies}

The ageing experiments are done in a self-made setup which resulted in a cooperative work with Heliatek GmbH (Dresden, Germany). This setup provides a long-term stable system for heating, illumination and measurement of multiple samples. Each sample can be individually heated to a certain temperature in the range from $40^{\circ} \mathrm{C}$ to approximately $100^{\circ} \mathrm{C}$. Since we generally have four identical devices on a $1 \mathrm{x} 1 \mathrm{inch}^{2}$ glass substrate we can apply different illumination intensities provided by two high power LEDs. Thus, it is possible to exchange the illumination colour between different experiments. White LEDs (Philipps Lumileds, LUXEON LXK2-PWC4-0220) with a continuous emission spectrum from 400 to $700 \mathrm{~nm}$ deliver up to $1000 \mathrm{~mW} / \mathrm{cm}^{2}$, which equals the intensity of ten suns, on the area of our devices $\left(6.44 \mathrm{~mm}^{2}\right)$. In addition to white illumination monochromatic LEDs are used to observe wavelength dependent ageing behaviour. In detail we illuminate at $461 \mathrm{~nm}$ (Philipps Lumileds, LUXEON LXHL-BB01) and $659 \mathrm{~nm}$ (Philipps Lumileds, LUXEON LXHL-BD01). Over time, the JV-curves are measured periodically to monitor the solar cell's characteristics.

\section{RESULTS AND DISCUSSION}

\subsection{The effect of illumination spectrum and atmospheric gases}

In this chapter, we show measurements of a substrate with four devices type A. All these solar cells have a mismatch and intensity corrected efficiency of $2.75 \%$.

For this experiment, the encapsulation was removed by pulling strain onto the glass and lifting the encapsulation lid with a scalpel. After this procedure, the sample was immediately attached to the ageing setup for a long-term measurement. Here, we used a blue (devices A1 and A2) and a red (A3, A4) LED with $470 \mathrm{~nm}$ and $617 \mathrm{~nm}$ peak-wavelength for illumination. In this experiment, the average temperature was $32 \pm 3{ }^{\circ} \mathrm{C}$ and the relative humidity $40 \pm 4 \%$.

The development of the normalized efficiency for all four devices is shown in Figure 1. The efficiency shows a rapid degradation for all four solar cells. Furthermore, it is obvious that the blue illumination (open symbols) leads to a faster faster degradation. For the first one, lifetimes of $43 \mathrm{~h}$ and $60 \mathrm{~h}$ can be observed. In this case, an increased intensity decreases the lifetime. The lifetime is defined as the time, when $80 \%$ of the initial efficiency is reached. The samples which are illuminated by the low-energy red illumination show substantially higher lifetimes of $95 \mathrm{~h}$ and $110 \mathrm{~h}$. 


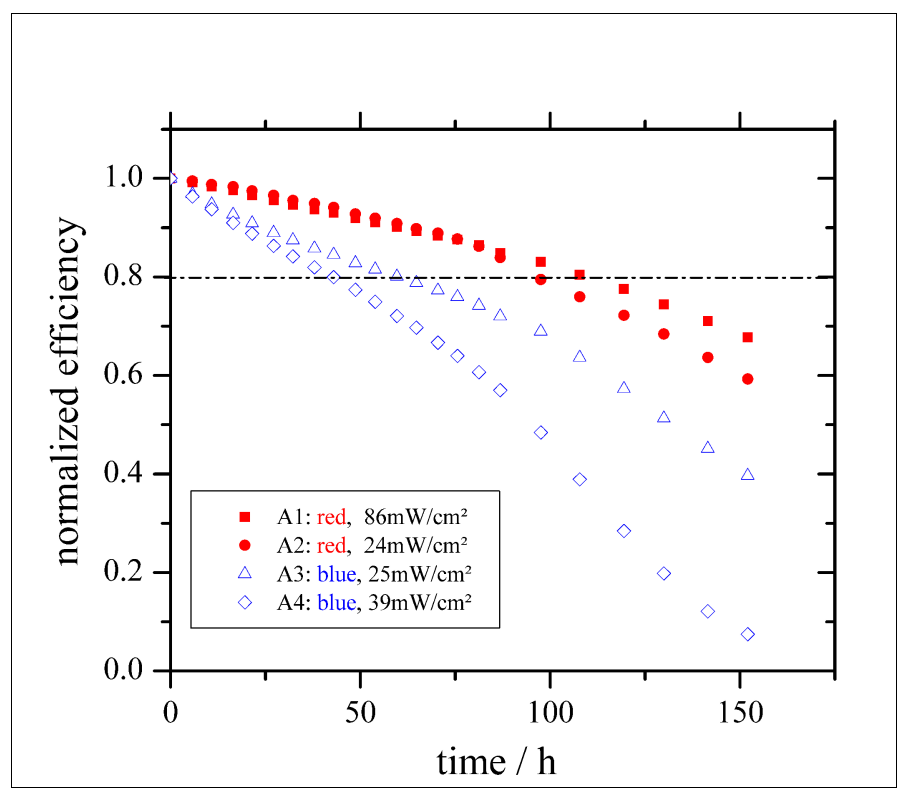

Figure 1: normalized efficiency over time for 4 identical devices without encapsulation; each two are illuminated with red and blue light; the dash-dotted line illustrates the point of $20 \%$ relative loss in efficiency, which generally defines the lifetime of a solar cell

However, the low-intensity device degrades faster for red illumination, which is unexpected. It turned out that sample A2 is close to $\mathrm{A} 3$ and is therefore exposed to at least a minor amount of blue light.

To identify the factors which determine the solar cell power conversion efficiency causes the degradation, we concentrate on the evolution of the IV-curves over the entire measurement time (see Figure 2). On the left side, for the blue illuminated device, the short-circuit-current $(86 \%$ loss in $155 \mathrm{~h})$ degrades as well as the Fill Factor (67\% relative loss) very strongly. The slower degradation of a red illuminated sample (right) is expressed in slight degradations in short-circuit-current and Fill Factor (20\% relative loss for each).

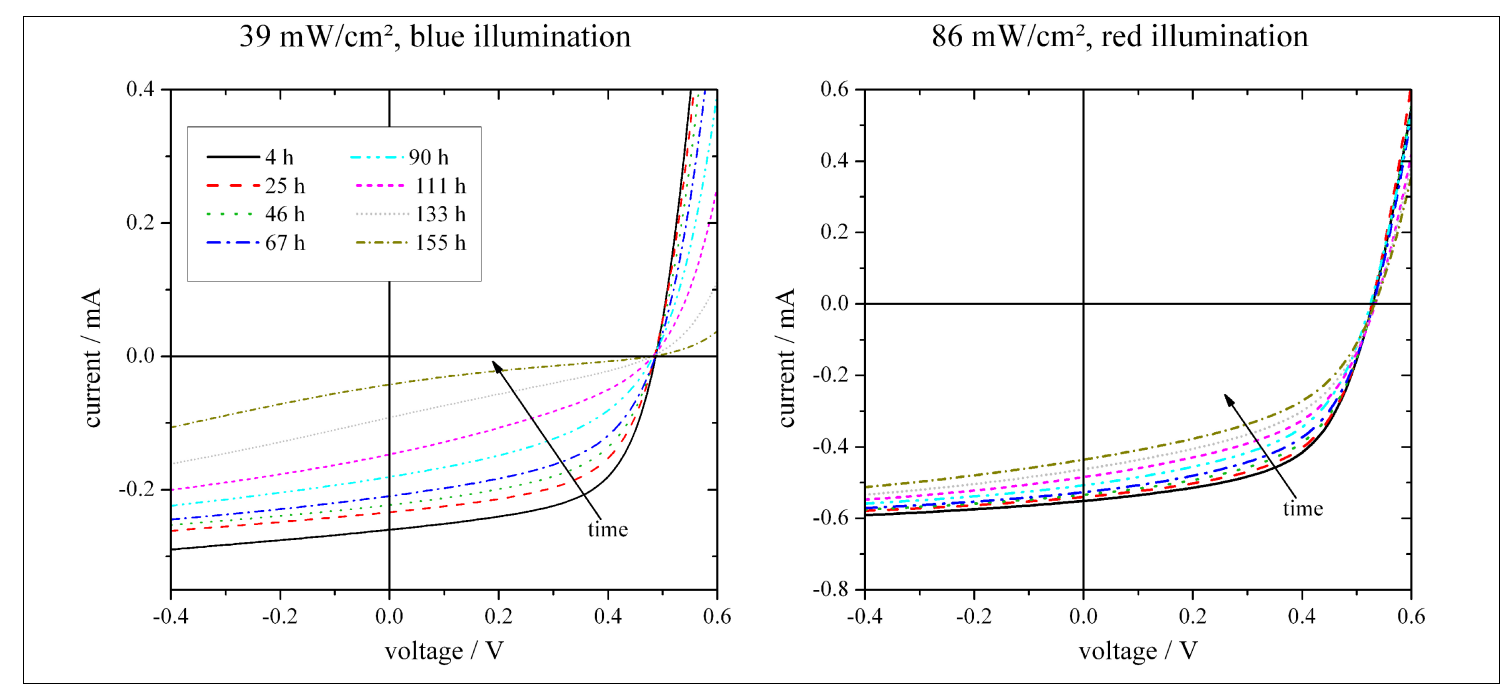

Figure 2: IV-curve evolution over time for devices under blue (left) and red (right) illumination without encapsulation 
One could argument that the losses in short circuit current are caused by a damage of the active materials which might be stronger for the high-energy $(2.7 \mathrm{eV})$ blue illumination. In addition to that, one should consider that the C60-absorption has a strong and broad peak around $450 \mathrm{~nm}$ and is therefore mostly excited under blue illumination whereas $\mathrm{ZnPc}$ contributes to the photocurrent in the range from $500 \mathrm{~nm}$ to $700 \mathrm{~nm}$ which correlates stronger to the emission spectrum of the red LED. To clarify this point, we measured the External Quantum Efficiency spectra of fresh and aged samples. The comparison can be seen in Figure 3.

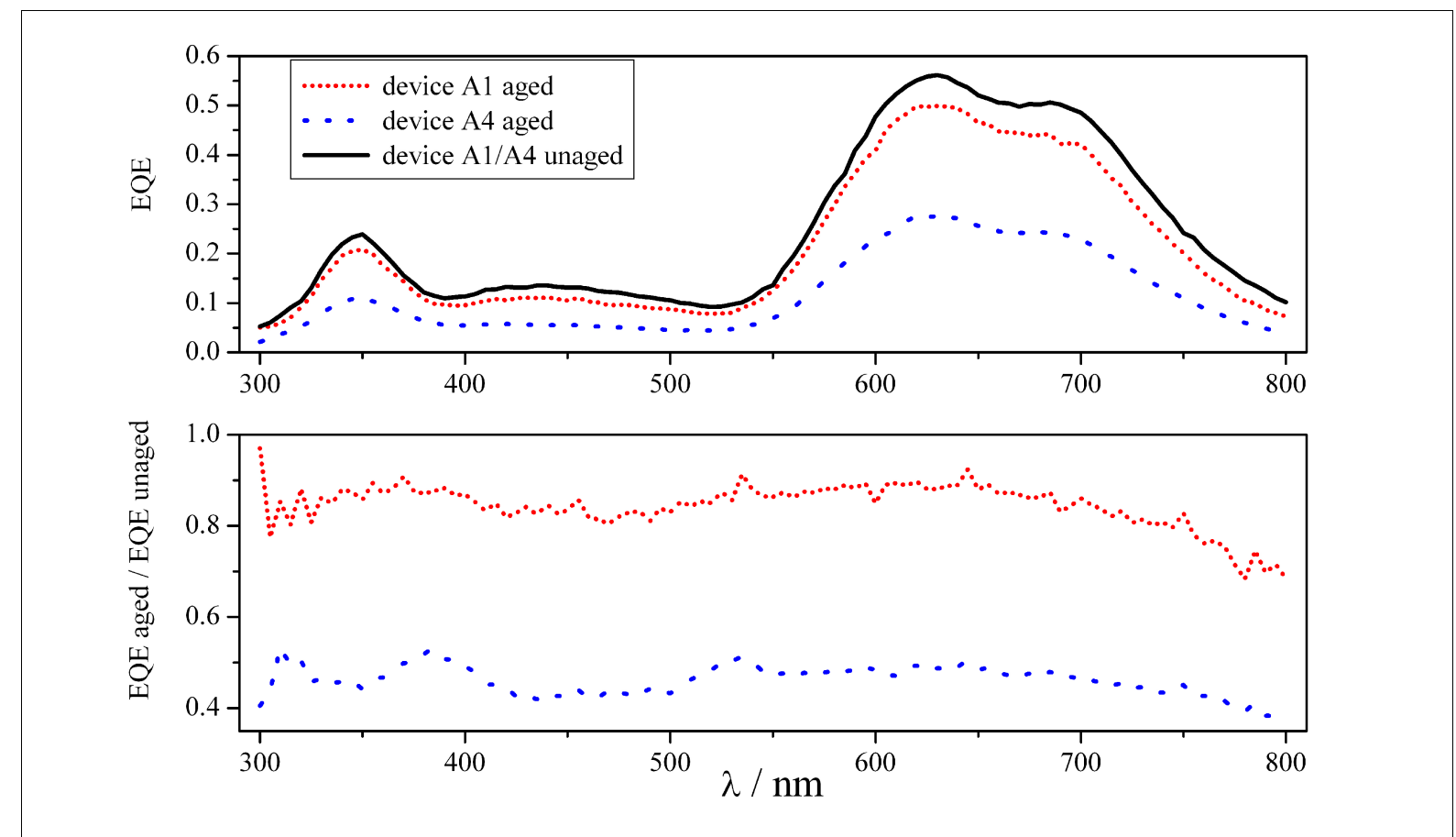

Figure 3: EQE data of the fresh and aged samples (top, A1 red illumination, A4 blue illumination); the lower graph illustrates the relative changes

As can be seen in figure 3, the blue illuminated device A4, which has a small short circuit current after degradation, shows a lower EQE than the red illuminated sample A1. The result in the lower part of Figure 3 shows that the relative shape of the EQE plot does not depend on the illumination wavelength. This is a clear evidence that the active materials $\mathrm{ZnPc}$ and $\mathrm{C} 60$ are stable even when they are strongly excited by monochromatic illumination. Therefore, the loss in device efficiency must be caused by changes in the transport layers or on an interface, e.g. the organic/aluminium or organic/ITO interface.

\subsection{The effect of temperature}

To figure out, whether the temperature has such a strong effect on degradation dynamics as reported in the literature, ${ }^{14,15}$ we aged three encapsulated samples of type A at different temperatures. The detailed conditions can be seen in table 1 . All devices were illuminated with comparable white emitting LEDs.

Table 1: Sample overview for temperature dependent ageing studies

\begin{tabular}{|c|c|c|}
\hline device & temperature $/{ }^{\circ} \mathbf{C}$ & intensity $/ \mathbf{~} \mathbf{W W} / \mathbf{c m}^{2}$ \\
\hline A5 & 50 & 10 \\
\hline A6 & 65 & 6 \\
\hline A7 & 95 & 39 \\
\hline
\end{tabular}


In figure 4, the normalized efficiency values of all three samples are plotted. The measurements at $50^{\circ} \mathrm{C}$ and $95^{\circ} \mathrm{C}$ lasted nearly $5500 \mathrm{~h}$, which is equivalent to 7.5 months. In this time, the sample at $50^{\circ} \mathrm{C}$ even improved its efficiency by $4 \%$ (relative) over the complete measurement time. At $65^{\circ} \mathrm{C}$, sample A6 slightly decreased its efficiency during the first $800 \mathrm{~h}$, followed by a decrease, leading to a final efficiency which was as good as the starting value. The most distinct

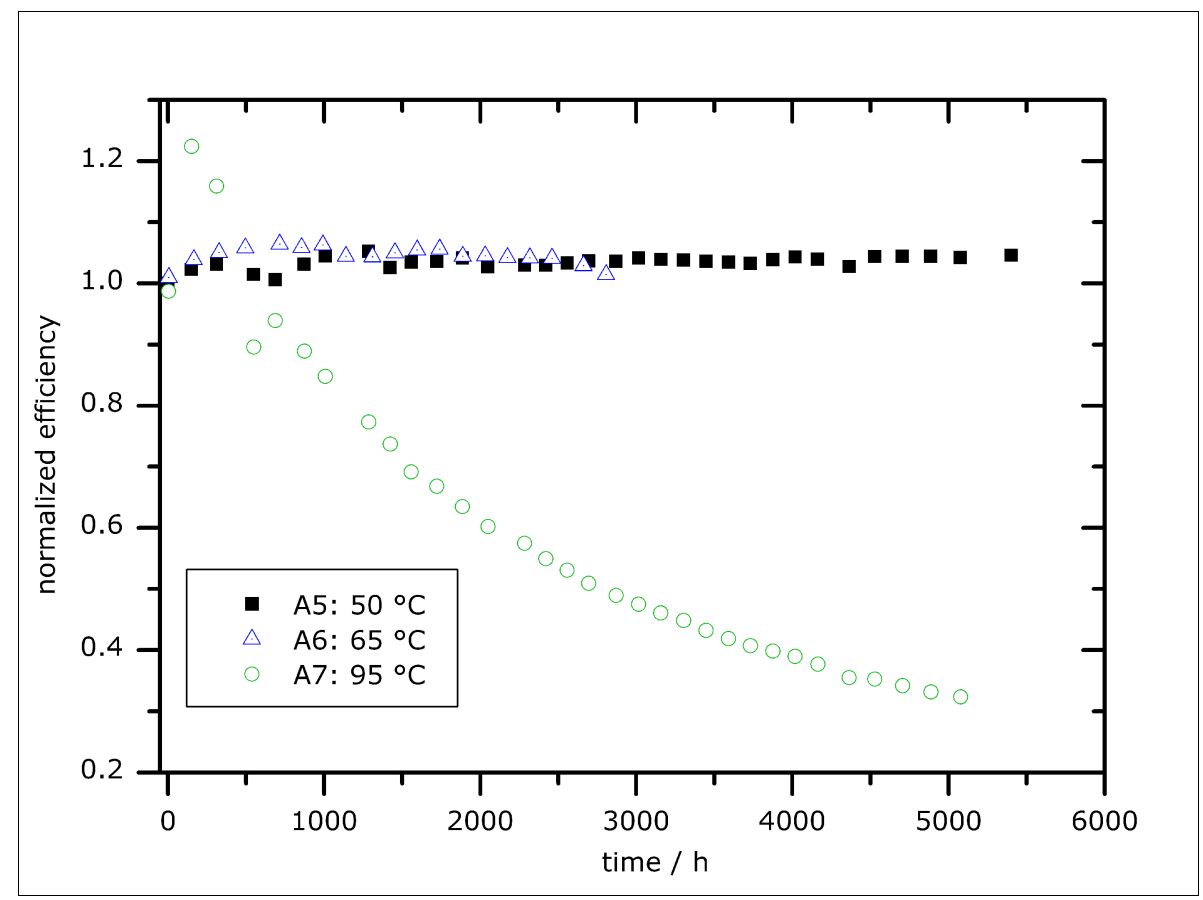

Figure 4: Ageing of three identical, encapsulated samples of type $\mathrm{A}$ at $50^{\circ} \mathrm{C}, 65^{\circ} \mathrm{C}$ and $95^{\circ} \mathrm{C}$

degradation which is shown in Figure 3 is the curve of sample A7, that was heated to $95^{\circ} \mathrm{C}$. This sample reaches a maximum in normalized efficiency after around $150 \mathrm{~h}$, resulting in a $22 \%$ higher value compared to the first value. After that, an exponential degradation behaviour is observed, causing a $70 \%$ loss after $5400 \mathrm{~h}$. At $95^{\circ} \mathrm{C}$ two different process seem to be present: On the one hand, a temperature activated annealing process enhances the overall efficiency during the first $150 \mathrm{~h}$. This enhancement is caused by open-circuit voltage, short-circuit current as well as the fill factor, each of them increased by $5 \%$ to $11 \%$. The second process, which causes the degradation over time, is a failure of encapsulation.

To analyse the temperature stability of our glass-glass encapsulation, we evaporated $60 \mathrm{~nm}$ thick calcium layers on identical substrates and used the standard encapsulation procedure. After that we stored some of these calcium samples at $25^{\circ} \mathrm{C}$ and $95^{\circ} \mathrm{C}$. Directly after production and later from time to time, we measured the transmission of these samples, using a Shimadzu UV3100.

At $95^{\circ} \mathrm{C}$, we measured a strong increase in transmission, starting at a value around $30 \%$ and reaching $93 \%$ after nearly $2000 \mathrm{~h}$. In contrast to that, the sample at room temperature showed a weaker increase form $30 \%$ to $55 \%$. To explain this we have to take into account that calcium easily reacts with water and oxygen molecules. ${ }^{18}$ Considering, that this reaction is slowed down at higher temperatures,${ }^{19}$ it is clear that we have a higher permeation of these molecules at increased temperatures. Supposing that each calcium atom reacts with one water molecule and extrapolating the area from the small edge of glue to the area of the complete organics, we calculate water vapour transmission rates (WVTR) of $3.5^{*} 10^{-4} \mathrm{gm}^{-2} \mathrm{~d}^{-1}$ at $95^{\circ} \mathrm{C}$ and $2.7 * 10^{-6} \mathrm{gm}^{-2} \mathrm{~d}^{-1}$ at room temperature.

In combination with the results of our solar cells, we conclude that our encapsulation shows a good barrier performance at temperatures between $25^{\circ} \mathrm{C}$ and $65^{\circ} \mathrm{C}$. In the range from $65^{\circ} \mathrm{C}$ to $95^{\circ} \mathrm{C}$, the WVTR increases by two orders of magnitude. This increased permeation of water (and perhaps oxygen) molecules leads to a chemical degradation of the solar cell performance, which causes extrinsic reactions. 


\subsection{The effect of (simulated) sunlight}

In this chapter, we show long-term measurement of a single device type A under continuous illumination of a sun simulator (Hoenle SOL $1200 \mathrm{MHG}$, Germany) with a medium intensity of $138 \pm 6 \mathrm{mWcm}^{-1}$. This sample was stored with encapsulation under a Nitrogen atmosphere, with oxygen and water concentrations below $1 \mathrm{ppm}$. In that case, we can be sure to analyse the intrinsic degradation caused by the illumination. The sample was actively cooled and had a temperature at about $30^{\circ} \mathrm{C}$ over the entire time.

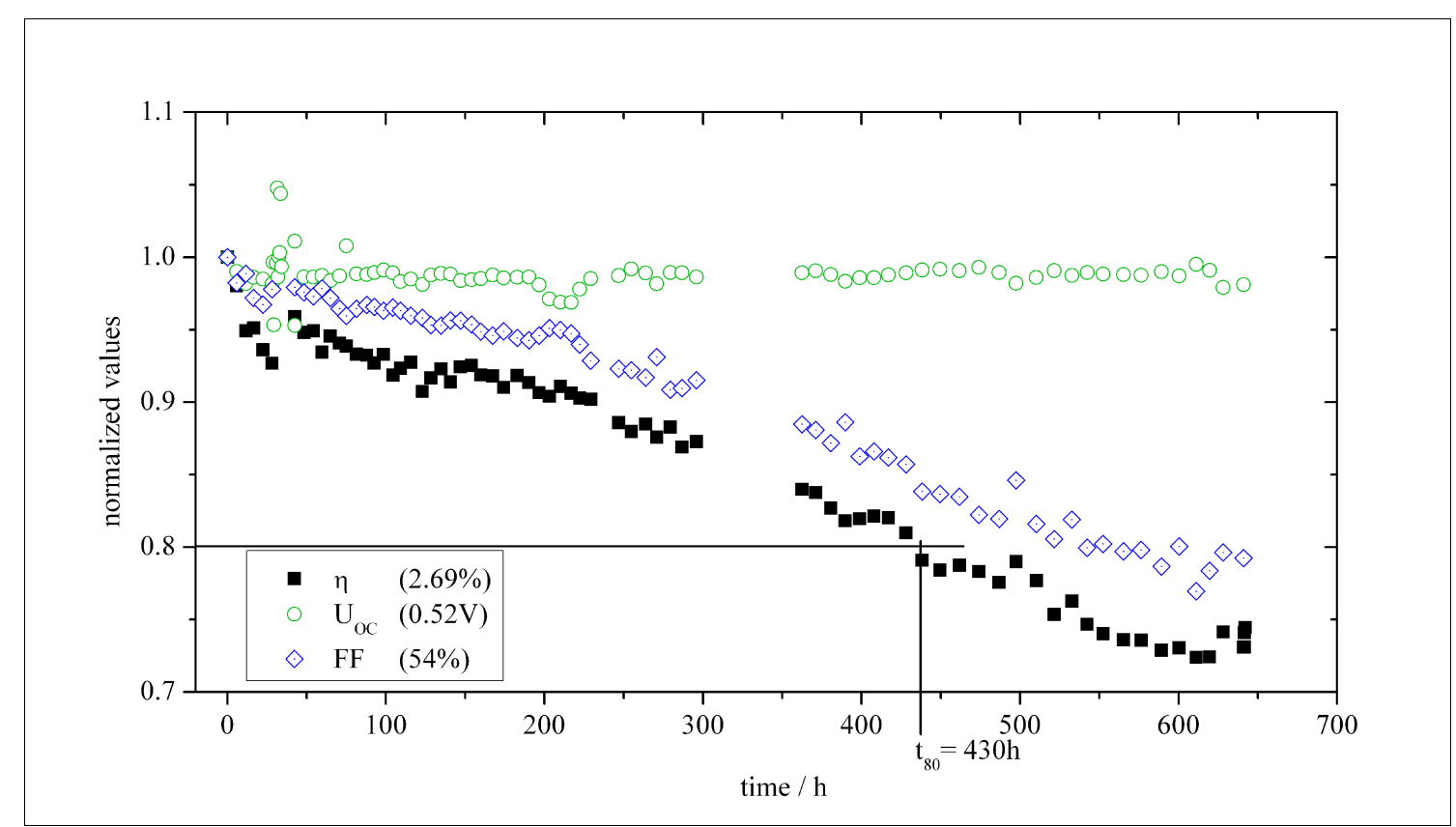

Figure 5: Evolution of basic electrical device parameters over time for an encapsulated device under illumination of a sun simulator; the number in brackets are the starting values of each quantity and the short circuit current density was around $9.6 \mathrm{~mA} / \mathrm{cm}^{2}$, corrected to $100 \mathrm{~mW} / \mathrm{cm}^{2}$

Due to high fluctuations up to $20 \%$, the short-circuit current density is not shown in this graph. Apart from these fluctuations, which are caused the intensity fluctuation of the sun simulator, no long-term trend is visible for $\mathrm{J}_{\mathrm{SC}}$. As the short-circuit current was not affected, the EQE did not change over time. This was proven by measurement, but is not shown here.

In figure 5 is the long-term behaviour of the three other important factors shown. As it is typical for our samples, the open-circuit voltage (open circles) does not show a systematic behaviour and remains constant. The slight drop after $200 \mathrm{~h}$ comes from a power loss of the fan which cools the sample. Again, this short heating period with temperatures which might be around $50^{\circ} \mathrm{C}$, does not show a long-term effect on the observed values. The continuous loss in fill factor is the driving force for the losses in efficiencies. As we did not see a comparable behaviour before, this can be related to the spectrum of the sun simulator, which contains ultra-violet radiation down to $300 \mathrm{~nm}$, contrary to the LEDs, which show emission from 400 to $800 \mathrm{~nm}$. This obviously damages at least one of our materials. As the fill factor is reduced (and not the short-circuit current density), we assume that neither $\mathrm{C} 60$ nor $\mathrm{ZnPc}$ is the damaged material. The most reasonable conclusion is that the hole-transport material Di-NPB is damaged, resulting in a worsened charge carrier transport and therefore reducing the fill factor. This leads to a short lifetime of $430 \mathrm{~h}$, which was similar for three other identical devices.

\subsection{The effect of illumination intensity}

After we figured out that the spectral shape of the illumination impacts a devices lifetime, we'll now have a look on the effect of different illumination intensities. For that, we set different intensities for the four solar cells on a substrate. All devices are of type B and will be named B1 to B4. The slightly different device structure compared to stack A is only due 


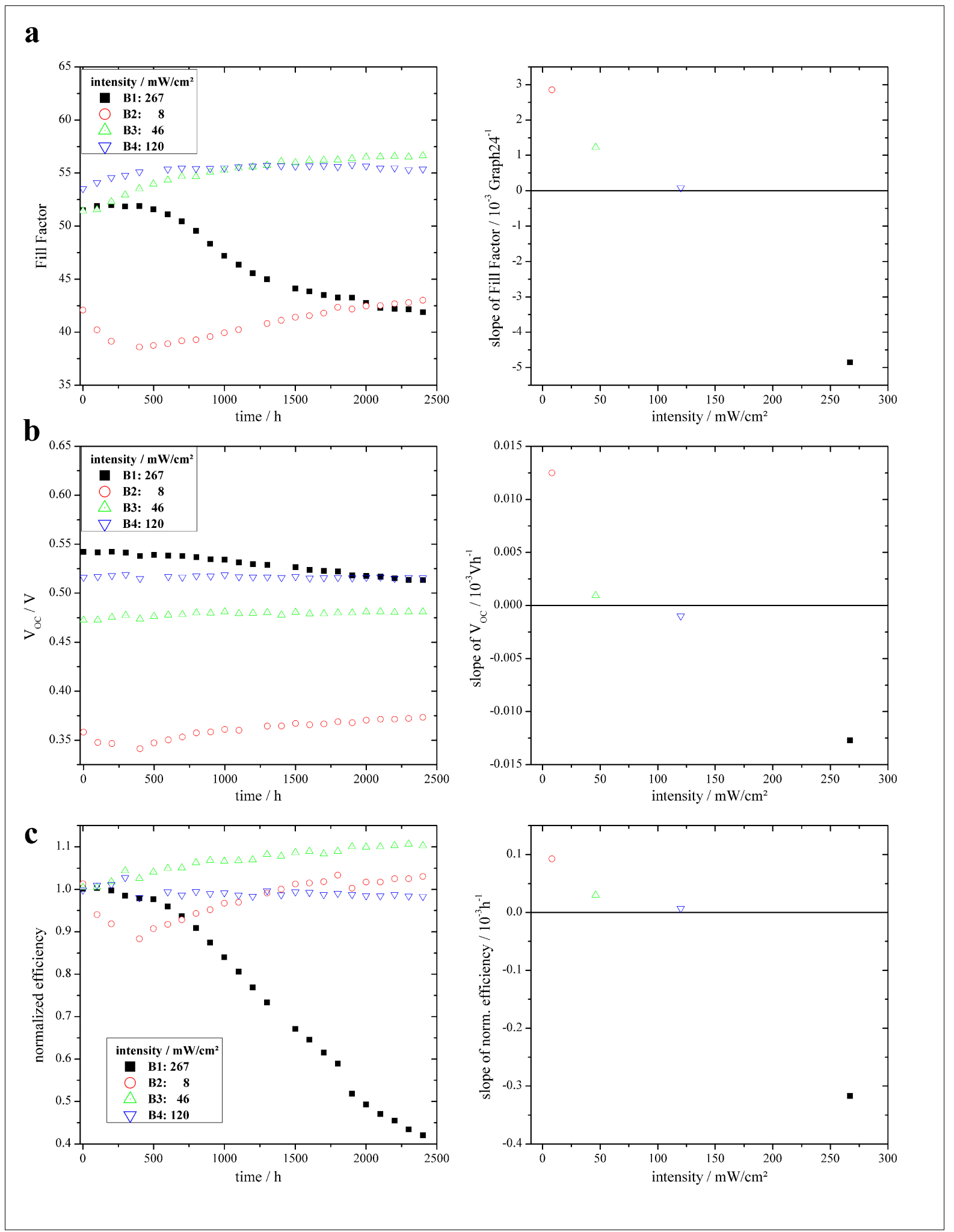

Figure 6: Evolution of fill factor (a), open-circuit voltage (b) and efficiency (c), on the left side. On the right side, the slope of each value for each cell is plotted over intensity. 
to processing reasons and should not affect device lifetime. Intensities from 8 to $267 \mathrm{~mW} / \mathrm{cm}^{2}$ are reached for this device. In figures 6a-c, the mainly affected device parameters fill factor (figure 6a), open-circuit voltage (figure 6b) and efficiency (figure 6c) are shown. In the left part of all pictures, each value is plotted over time. The right one shows the degradation slope of the values over the illumination intensity. Due to non-linear effects during the first 1000 hours, the slope is calculated for the time span from 1000 to 1800 hours. Despite slight variations, she short-circuit current density remains unaffected over the entire measurement range and will be neglected in the following analysis.

For the strongest illuminated device (black squares, $267 \mathrm{~mW} / \mathrm{cm}^{2}$ ) we see losses in all three parameters over the entire measurement time of 2500 hours. This leads to the data point in the lower right of each graph on the right side, expressed by a negative slope of all parameters. Going down to an intensity a bit higher than one sun (down triangle, $120 \mathrm{~mW} / \mathrm{cm}^{2}$ ), the slight improvement of the fill factor is nearly balanced by the loss in open-circuit voltage, resulting in an almost stable efficiency over time. By illuminating lower intensities, both device even improve over time.

One can also see from these graphs that the fill factor reaches its best value at an intensity of approximately one sun, whereas illuminating more or less light reduces that value. As it is well known from literature the open-circuit voltage is influenced by charge carrier density, which increases with stronger illumination. This behaviour is also visible in our measurements.

\section{CONCLUSIONS}

In summary, we showed that the selection of ageing conditions has a major impact on the resulting degradation dynamics of small molecule organic solar cells. In contrast to the results of Schuller et al. we did not see an impact of temperature on the ageing of encapsulated results. ${ }^{15}$ As long as the encapsulation works, the devices show constant efficiencies over more than 5000 hours. Increasing the temperature up to $95^{\circ} \mathrm{C}$, the glass-glass encapsulation shows an increased WVTR (and probably also an increased Oxygen Transmission Rate (OTR)), which leads to device degradation.

Comparing different monochromatic illuminations, higher photon energies accelerate the ageing compared to lower energy radiation. However, the relative shape of the EQE spectra show no variations over time. This is a clear evidence for the stability of our active materials ZnPc and C60. This is in contrast to the results of Lessmann et al. where the oxidation C60 is strongly expressed in a relative change of the EQE. ${ }^{14}$ Using a sun simulator as light source leads to a systematic decrease of the fill factor, most likely caused by damages in the hole transport material.

The comparison of different illumination intensities validates the stability of the observed solar cells at moderate stress conditions. It takes more than two suns to get a noticeable degradation of a $60 \%$ loss in efficiency over 2500 hours.

Future studies must be done to compare different measurement conditions, also taking into account that there might be differences for different materials, e.g. small molecules vs. polymers. There is a need for the understanding of the underlying degradation processes to further increase the stability of small molecule organic solar cells, which is already on a high level.

\section{ACKNOWLEDGEMENTS}

This work is funded by the Bundesministerium für Bildung und Forschung in the framework of the InnoProfile project (03IP602). 


\section{REFERENCES}

[1] Martin A. Green, Keith Emery, Yoshihiro Hishikawa and Wilhelm Warta, "Solar cell efficiency tables (version 35)", Prog. Photovolt: Res. Appl. 2010, 18, 144-150 (2010)

[2] Riede, M. K., Schueppel, R., Schulze, K., Wynands, D., Timmreck, R., Uhrich, C., Petrich, A., Pfeiffer, M., Brier, E., Reinold, E., Baeuerle, P. \& Leo, K. Gombert, A. (ed.), "Recent progress in organic solar cells based on small molecules", Photonics for Solar Energy Systems II, 7002, 70020G (2008)

[3] Used for better processibility, comparable in performance to the commonly available 2,3,5,6-tetrafluoro-7,7,8,8tetracyanoquinodimethane (F4-TCNQ).

[4] Peter Peumans, Aharon Yakimov, and Stephen R. Forrest "Small molecular weight organic thin-film photodetectors and solar cells", Journal of Applied Physics, 93(7), 3693-3723 (2003)

[5] Used for better processibility, comparable in performance to the commonly available acridine orange base (AOB).

[6] Jørgensen, K. Norman and F. Krebs, "Stability / degradation of organic solar cells", Solar Energy Materials \& Solar Cells 92, 686-714 (2008)

[7] Q.L. Song et al., "Small-molecule organic solar cells with improved stability", Chemical Physics Letters 416, 42-46 (2005)

[8] Q. L. Song et al., "Degradation of small-molecule organic solar cells", Applied Physics Letters 89, 251118 ( 2006)

[9] $\quad \mathrm{H}$. Wu et al., "Stable small-molecule organic solar cells with 1,3,5-tris(2-n-phenylbenzimidazolyl) benzene as an organic buffer", Thin Solid Films 515, 8050-8053 (2007)

[10] H. Shiokawa, M. Yokoyama and M. Hiramoto, "Long-term operation tests of organic solid-state solar cells", Surface Review \& Letters 14, 539-542 (2007)

[11] P. Vivo, J. Jukola, M. Ojala, V. Chukharev and H. Lemmetyinen, "Influence of Alq3/Au cathode on stability and efficiency of a layered organic solar cell in air", Solar energy materials and solar cells 92, 1416-1420 (2008)

[12] P. Sullivan and T. Jones, "Pentacene/fullerene (C60) heterojunction solar cells: Device performance and degradation mechanisms", Organic Electronics 9, 656-660 (2008)

[13] R. Franke, B. Maennig, A. Petrich and M. Pfeiffer, "Long-term stability of tandem solar cells containing small organic molecules", Solar energy materials and solar cells 92, Issue 7, 732-735 (2008)

[14] R. Lessmann, Z. Hong, S. Scholz, B. Maennig M.K. Riede and K.Leo, "Aging of flat heterojunction phthalocynine/fullerene C60 organic solar cells", Organic Electronics 11, 539-543 (2010)

[15] S. Schuller, P. Schilinsky, J. Hauch and C. Brabec, "Determination of the degradation constant of bulk heterojunction solar cells by accelerated lifetime measurements", Applied Physics A - Materials Science \& Processing, 79, 37-40 (2004)

[16] Suren A. Gevorgyan, Mikkel Jørgensen and Frederik C. Krebs, "A setup for studying stability and degradation of polymer solar cells“, Solar Energy Materials and Solar Cells, 92, Issue 7, 736-745 (2008)

[17] http://konarka.com/

[18] R. Paetzold, A. Winnacker, D. Henseler, V. Cesauri and K. Heuser, "Permeation rate measurements by electrical analysis of calcium corrosion", Review of Scientific Instruments, 74, 5147-5150 (2003)

[19] D. Nissen, "Low-temperature oxidation of calcium by water-vapor", Oxidation of metals 11, 241-261 (1977)

[20] V. Shrotriya, G. Li, Y. Yao, T. Moriarty, K. Emery and Y. Yang, "Accurate Measurement and Characterization of Organic Solar Cells”, Advanced Functional Materials, 16, Issue 16, 2016-2023 (2006) 